\title{
Synthesis and Characterization of Agnps from Vitis Vinifera Peel Extract and Its Antimicrobial Efficacy
}

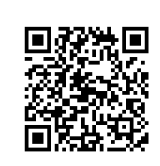

\author{
Divya R, Supraja N and David E* \\ Department of Biotechnology, Thiruvalluvar University, vellore, serkkadu- 632001, India \\ *Corresponding author: David E, Department of Biotechnology, Thiruvalluvar University, vellore, serkkadu- 632001,India
}

Submission: November 30, 2018; Published: January 17, 2019

\begin{abstract}
A green synthesis of silver nitrate nanoparticles was carried out using Vitis vinifera skin peel extract. Synthesized nanoparticles were characterized using UV-Vis absorption spectroscopy, DLS, TEM, FT-IR and XRD studies respectively. UV-Vis absorption spectroscopy of prepared silver nitrate colloidal solution showed absorption maxima at $360 \mathrm{~nm}$. TEM and analysis showed average particle size ranges from $0.2-0.5 \mu \mathrm{m}$ whereas SEM shown $400 \mu \mathrm{m}$, while XRD confirms SAED pattern confirmed the crystalline nature of synthesized nanoparticles. FT-IR analysis indicated the involvement of hydroxyl $(\mathrm{O}-\mathrm{H})$, nitrates, carboxylic acids and amine $(\mathrm{N}-\mathrm{H})$ functional groups of Vitis vinifera peel extract in preparation of silver nitrate nanoparticles. DLS shows the particle size of silver nitrate nanoparticles is $91.6 \mathrm{~nm}$ and zeta potential is $-20.7 \mathrm{mV}$. Hence from this study we concluded that the nanoparticles synthesized from Vitis vinifera peel extract has shown very effective on in-vitro antimicrobial activity (Gram positive and negative Bactria, Fungi) using disc diffusion method.
\end{abstract}

Keywords: Vitis vinifera; Silver nanoparticles; Antimicrobial

\section{Introduction}

Particles with a size up to $100 \mathrm{~nm}$ are usually referred to as nanoparticles. Nanoparticles exhibit completely new or improved properties, based on specific characteristics, such as grain size, distribution, morphology, and higher surface to volume ratio if compared with larger particles of the bulk material. A specific surface area is relevant to catalytic reactivity and other related properties, such as antimicrobial activity in silver nanoparticles (AgNPs). The prefix "nano" has found much interest in last decades as an increasing application to different fields of the knowledge. Nano science, nanotechnology, nano materials, nano chemistry are some of few nano-containing terms that occur frequently in scientific reports, in popular books and also in newspapers and that have become familiar. Nanotechnology is an important field of modern scientific research that deals with synthesis, strategy and manipulation of particles structure ranging from approximately 1 to $100 \mathrm{~nm}$ in size [1]. At this size, the atoms and molecules work differently in a dynamic way due to the change in physical chemical and biological properties. These dynamic changes provide a variety of surprising and interesting uses [2]. It provides opportunities for the development of materials that includes medical and pharmaceutical applications due to its boosting properties of materials in the form of nanosized particles. The development of green processes for the synthesis of nanoparticles has been evolving into an important branch of nanotechnology as green nanotechnology that deals with the safer and eco-friendly methods for nano materials fabrication and which is considered as an alternative to conventional physical and chemical methods [3]. Production of NPs may be achieved through different methods. Chemical methods are the most popular methods to produce NPs. However, chemical methods cannot evade the use of toxic chemicals in the synthesis protocol. Since noble metal NPs, such as gold, silver, and platinum, are widely applied to human contact areas, there is a growing need to develop environmentally benign protocol of NPs synthesis that avoids the use of toxic chemicals. Biological methods of NPs synthesis using bacteria, fungi, plants, algae, sea weed, and lichen have been recommended as ecofriendly alternatives to chemical and physical process.

In this paper, we present a simple and rapid biosynthesis of silver nanoparticles using Vitis vinifera peel extract. As prepared, Ag nanoparticles were characterized by various methods, such as UV-Vis, FT-IR, DLS, SEM, TEM and XRD for antimicrobial activity. This work provided a potential approach for the production of Ag nanoparticles without the involvement of additional chemicals and physical steps.

\section{Materials and Methods}

\section{Biosynthesis of silver nanoparticles}

Vitis vinifera peel was collected from Acharya N G Ranga Agricultural University, Tirupati (Figure 1). The peel were airdried for 10 days, then kept in a hot air oven at $50{ }^{\circ} \mathrm{C}$ for $24 \mathrm{~h}$. peel were ground to fine powder. $10 \mathrm{~g}$ of powder was mixed in $100 \mathrm{ml}$ of water and boiled at $85{ }^{\circ} \mathrm{C}$ for $90 \mathrm{~min}$ and the solution was filtered 
using Whatman filter paper (No.1) and the extract was collected and stored in a plastic bottle. $90 \mathrm{ml}$ of $\mathrm{mM}$ silver nitrate is added to $10 \mathrm{ml}$ of extract and incubated for one day. A change in the color of solution was observed it indicates the formation of nanoparticles [4].

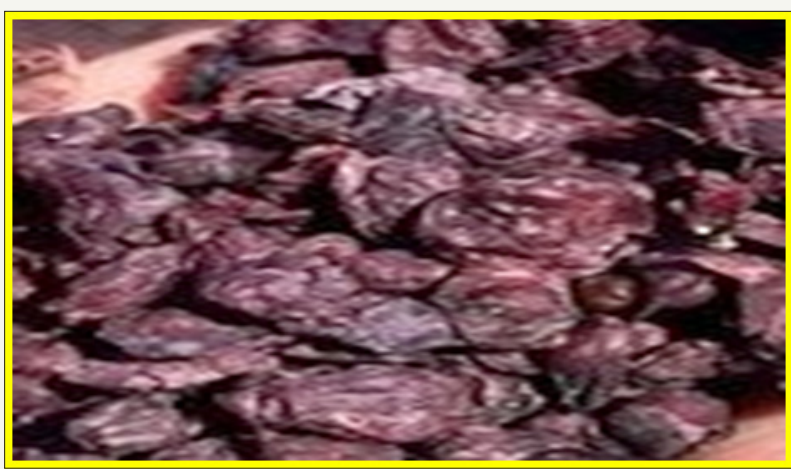

Figure 1: Vitis vinifera peel.

\section{Collection of microbes (Bacteria and fungi)}

The microbes (Bacteria and fungi) samples were collected from Nanotechnology laboratory, Regional Agricultural Research Station, Tirupathi, (Chittoor District) Andhra Pradesh, India. These samples were stored in an ice box and transported to the laboratory for microbiological characterization. Through serial dilution pour plate technique, fungal sp. was isolated using potato dextrose agar (PDA) medium, and Gram negative and Gram-positive bacteria were isolated from nutrient agar medium. Further, it is maintained in potato dextrose agar slants (fungi) and nutrient agar slants (bacteria) for onward analysis.

\section{Antibacterial activity}

The antibacterial activity of AgNPs was evaluated against the pathogenic strains E. coli, Pseudomonas, Staphylococcus aureus, Klebsilia, Salmonella typhimurium and Bacillus pantothenticus. These cultures were grown in appropriate mediums at $37{ }^{\circ} \mathrm{C}$ for overnight incubation and maintained at $4{ }^{\circ} \mathrm{C}$ in a refrigerator. Disc (for disc diffusion method) of $5 \mathrm{~mm}$ was made for nutrient agar medium and each disc was dipped at different concentrations $(170,100,50 \mathrm{ppm})$ of prepared AgNPs. The pure cultures of bacterial pathogens were sub-cultured in an appropriate medium. After incubation at $37{ }^{\circ} \mathrm{C}$ for $24 \mathrm{~h}$ the bacterial inhibition zones were calculated. These assays were performed in triplicate [5].

\section{Antifungal activity of Gracilaria corticata produced Ag- NPs}

The antibacterial activity of AgNPs was evaluated against the pathogenic strains Aspergilus niger, Aspergilus flavus, Meyerozyma caribbica, Hypocrea koningii, Fusarium oxysporium and Rhizopus stolonifer. These cultures were grown in appropriate medium at 37 ${ }^{\circ} \mathrm{C}$ for overnight incubation and maintained at $4{ }^{\circ} \mathrm{C}$ in a refrigerator. Disc of $5 \mathrm{~mm}$ was made on nutrient agar medium and each disc was dipped at different concentrations $(170,100,50 \mathrm{ppm})$ of AgNPs. The pure cultures of fungal pathogens were sub cultured in an appropriate medium and each strain was swabbed uniformly on to the individual plate. After incubation at $37^{\circ} \mathrm{C}$ for $24 \mathrm{~h}$ the inhibition zones were measured in triplicate [6].

\section{Characterization of Ag nanoparticles}

Nanoscale particles exhibit the localized surface plasmon resonance (LSPR) phenomenon which represents the characteristic absorption spectra of the nanoparticles (formed silver nanoparticles) and was recorded using UV-Vis Spectrophotometer (50 ANALYTIKJENA) run in the wavelength range from 250 to $400 \mathrm{~nm}$. The FT-IR spectrum of the sample containing silver nanoparticles was recorded by directly placing $\mathrm{KBr}$ crystal using ATR (attenuated total reflectance) technique in the transmittance mode and run in the mid IR region of $400-4000 \mathrm{~cm}^{-1}$. X-ray diffraction technique was used to reveal the crystal structure of the prepared silver nanoparticles. Model: JPX-8030) with $\mathrm{CuK}$ radiation (Ni filtered $=13418 \mathrm{~A}$ ) at $40 \mathrm{kV}, 20 \mathrm{~A}$. The hydrosol was filtered through a $0.22 \mu \mathrm{m}$ syringe driven filter unit and the size (hydrodynamic diameter) and distribution were measured by using the Dynamic Light Scattering (DLS) technique (Nanopartica, HORIBA, SZ-100). The surface morphological studies including size and shape of the formed silver nanoparticles were carried out by analyzing the samples under scanning electron microscope (Hitachi's SU6600) operated at accelerating voltage of $30 \mathrm{Kv}$ (with the magnification ranging from $10 \times$ to 600,000$)$, TEM (H-7100 Hitachi).

\section{Results and Discussion}

\section{UV-visible spectrum}

In this study, extract when interacting with the silver nitrate salt solution form a dark brown solution due to the reduction of the silver ion to AgNPs followed by a colour change indicating the biotransformation of ionic silver to reduced silver and the subsequent formation of AgNPs in an aqueous medium. The colour change was monitored visually and the peak at $360 \mathrm{~nm}$ in the UVvisible spectra indicated the presence of AgNPs which may be due to the excitation of surface plasmon vibrations in AgNPs (Figure 2).

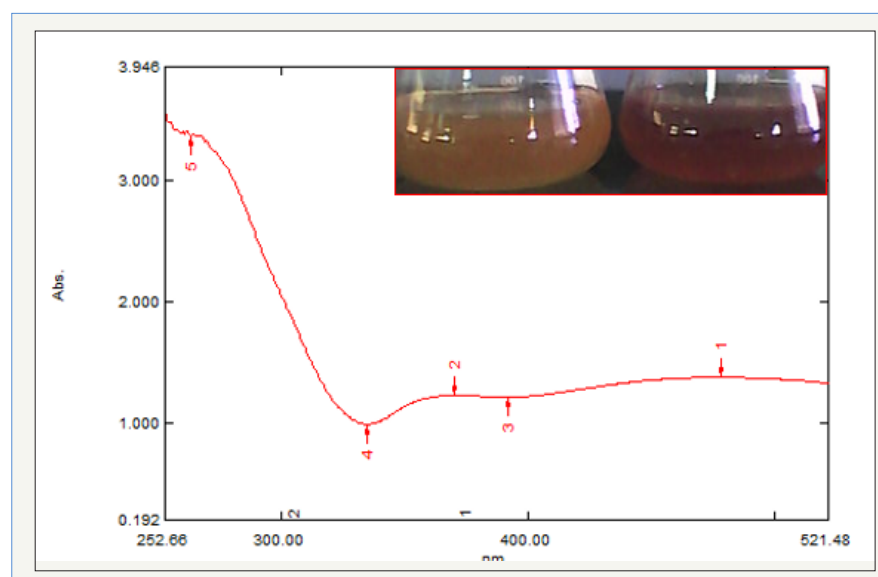

Figure 1: Showing UV-visible spectrum of Vitis vinifera peel extract mediated synthesized AgNPs.

\section{FT-IR spectrum}

The FT-IR spectrum shows the presence of band at 3356, 2886, $2818,2102,1637,1406$, and $580 \mathrm{~cm}^{-1}$ corresponding to monosubstituted amide, nitro, primary amide, carboxylic, and alcohol 
group, respectively. The band at 3356 and $2886 \mathrm{~cm}^{-1}$ is due to methylene scissoring vibrations present in the proteins. Largely, the observation confirms the presence of protein in AgNPs. 2818 and 2102 indicating presence of carboxylic groups, 1637 and 1406 indicating the presence of nitriles groups and 580 indicating the presence of alkyl halides group (Figure 3), FT-IR spectroscopic study has confirmed that the mono substituted amide of proteins has the stronger ability to bind metal, this data suggests that the biological molecules could probably perform the reduction and stabilization of the AgNPs [7].

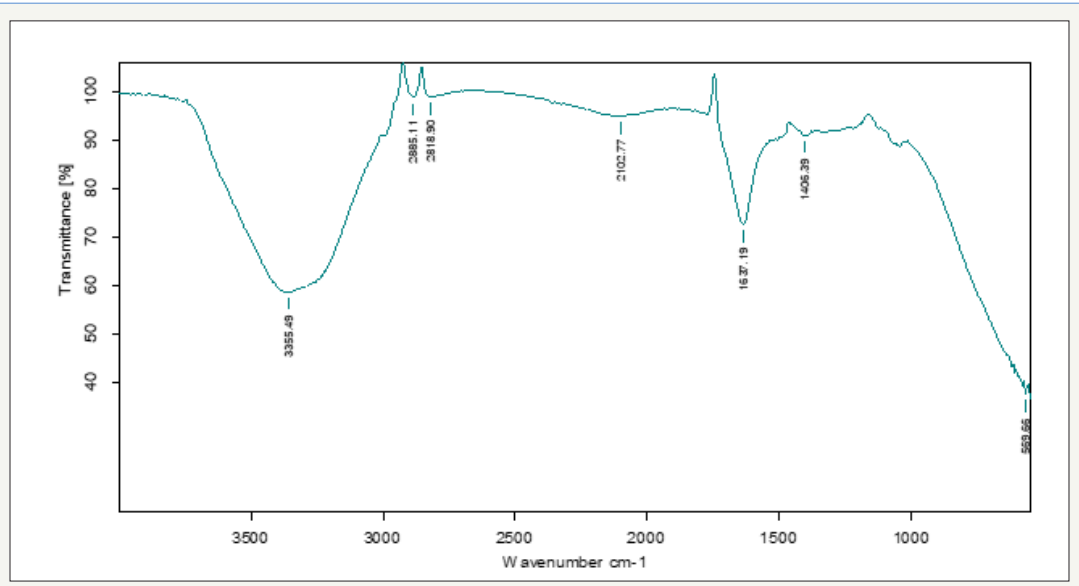

Figure 3: Showing FT-IR spectrum of Vitis vinifera peel extract mediated synthesized AgNPs.

\section{X-Ray diffraction}

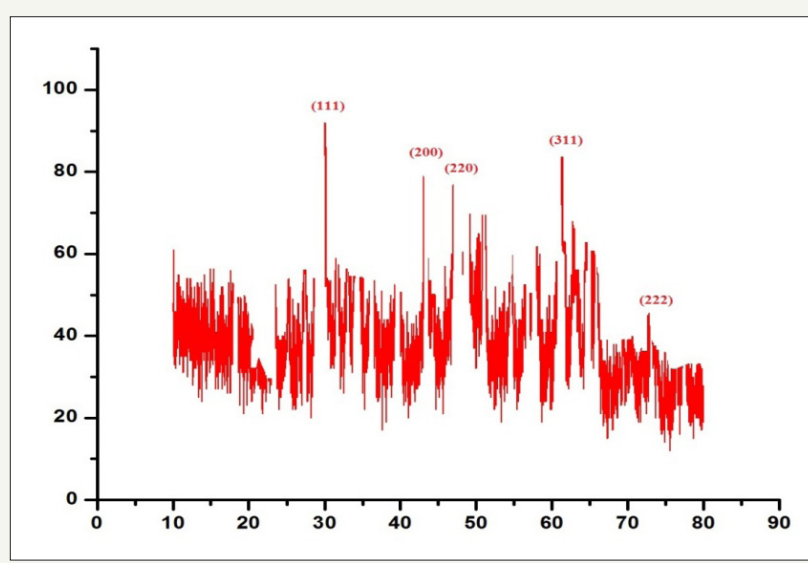

Figure 4: Showing XRD of Vitis vinifera peel extract mediated synthesized AgNPs.
The XRD pattern of the AgNPs is shown in Figure 4. Bragg reflections obtained in the micrograph clearly indicated the presence of (111), (200), (220), (311) and (222) sets of lattice planes which is a consequence of crystalline nature of formed AgNPs and indexed as face-centered-cubic (FCC) structure of silver. In addition to the Bragg peaks representative of FCC AgNPs, additional as yet unassigned peaks are also observed suggesting that the crystallization of bio-organic phase occurs on the surface of the nanoparticles.

\section{Dynamic light scattering analysis}

The hydrodynamic diameter (size) of the AgNPs was found to be $91.6 \mathrm{~nm}$ (Figure 5a) and was measured as a function of scattering angle of the laser from the surface of the particle. Further, zeta potential of AgNPs was also measured and was recorded as $20.7 \mathrm{mV}$ (Figure $5 \mathrm{~b}$ ). The zeta potential clearly indicates stability of the prepared AgNPs.

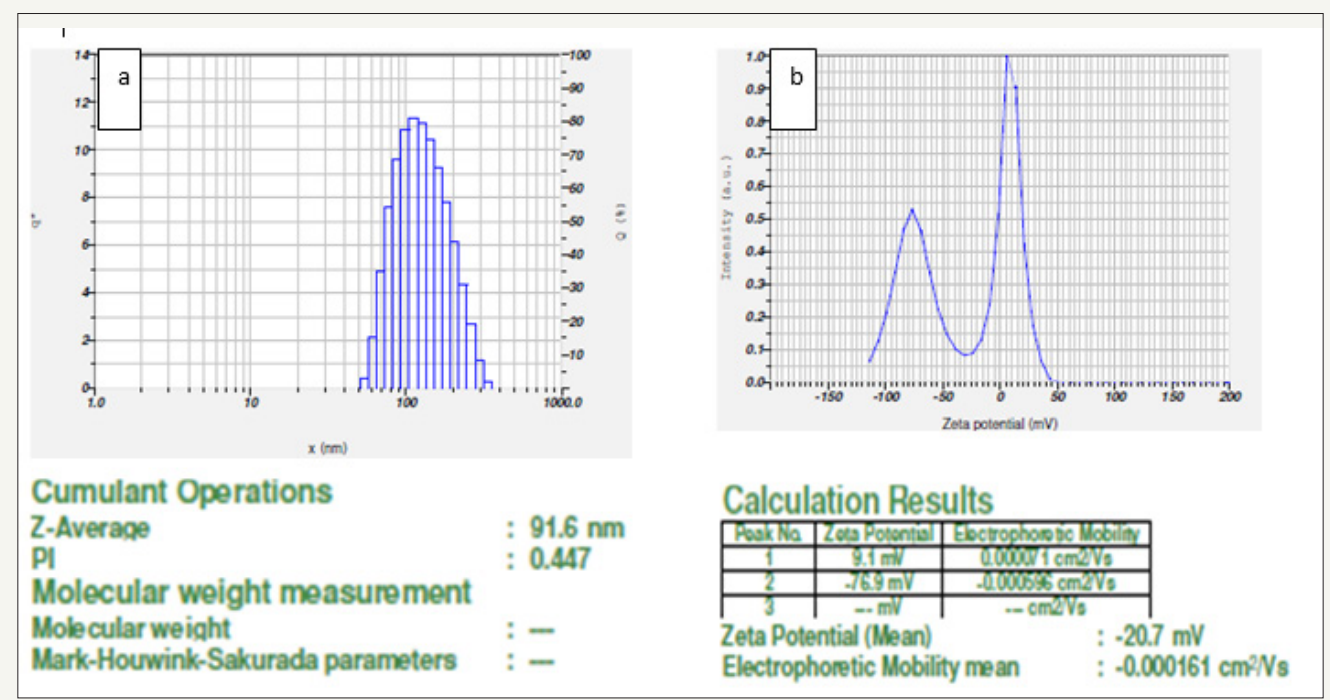

Figure 5(a\&zb): Particle size and showing zeta potential of Vitis vinifera peel extract mediated synthesized AgNPs. 


\section{Scanning electron microscopy analysis}

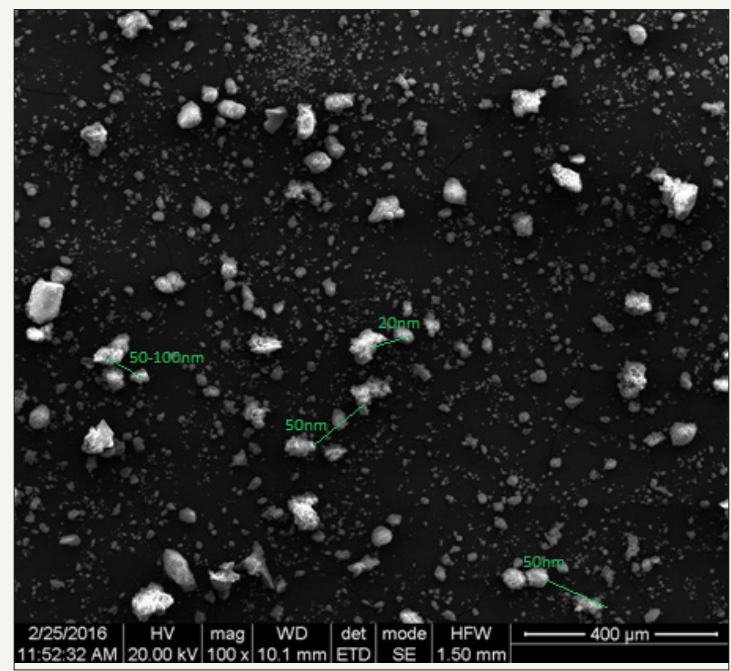

Figure 6: Showing SEM analysis of Vitis vinifera peel extract mediated synthesized AgNPs.

Surface morphology of silver nanoparticles was studied from the SEM micrograph. It is evident that AgNPs were spherical in shape and were poly-dispersed. The measured average size of AgNPs was $400 \mu \mathrm{m}$ (Figure 6).

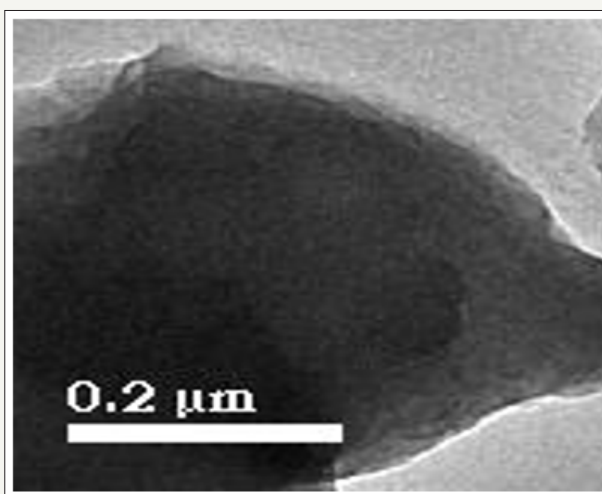

\section{Transmission Electron Microscopy}

The TEM images showed that most of the particles are irregular and agglomerated spherical in shape and created big agglomerates which indicated the monodispersed nature of NPs. The TEM images revealed that AgNPs are in the range of 20-50 $\mu \mathrm{m}$ (Figure 7).

Figure 7: Showing TEM analysis of Vitis vinifera peel extract mediated synthesized AgNPs.

\section{Antimicrobial assay}

It is well-known that silver nanoparticles exhibit brown color, arising due to excitation of

surface Plasmon vibrations in the silver nanoparticles. Silver nanoparticles shown have very strong inhibitory action against

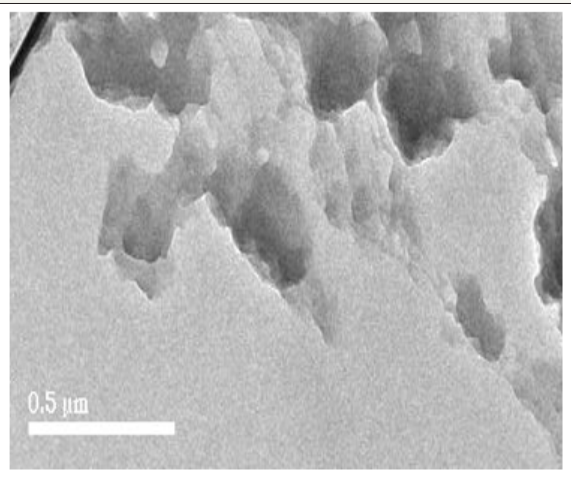

Table 1: Antibacterial zone of inhibition and Standard Deviation of Vitis vinifera peel extract mediated synthesized AgNPs.

\begin{tabular}{|c|c|c|c|c|}
\hline S. No & Bacteria Names & $\mathbf{1 7 0 p p m} \pm \mathbf{1 . 4 p p m}$ & $\mathbf{1 0 0} \mathbf{p p m} \pm \mathbf{1 . 1} \mathbf{p p m}$ & $\mathbf{5 0 p p m} \pm \mathbf{0 . 8 p p m}$ \\
\hline 1 & Bacillus pantothenticus & $1.2 \pm 0.07$ & $0.8 \pm 0.05$ & $0.5 \pm 0.03$ \\
\hline 2 & Escherichia Coli & $1.9 \pm 0.08$ & $1.4 \pm 0.06$ & $1.1 \pm 0.02$ \\
\hline 3 & Pseudomonas & $1.7 \pm 0.1$ & $1.4 \pm 0.09$ & $0.9 \pm 0.07$ \\
\hline 4 & Salmonella typhimurium & $2.8 \pm 0.4$ & $1.9 \pm 0.2$ & $1.5 \pm 0.09$ \\
\hline 5 & Staphylococcus aureus & $3.4 \pm 0.5$ & $2.7 \pm 0.3$ & $2.2 \pm 0.1$ \\
\hline 6 & Klebsilia & $2.9 \pm 0.1$ & $2.1 \pm 0.09$ & $1.5 \pm 0.06$ \\
\hline
\end{tabular}


Table 2: Antifungal zone of inhibition and Standard Deviation of Vitis vinifera peel extract mediated synthesized AgNPs.

\begin{tabular}{|c|c|c|c|c|}
\hline S. No & Fungi Names & $\mathbf{1 7 0} \mathbf{p p m} \pm \mathbf{1 . 4} \mathbf{p p m}$ & $\mathbf{1 0 0} \mathbf{p p m} \pm \mathbf{1 . 1} \mathbf{p p m}$ & $\mathbf{5 0} \mathbf{p p m} \pm \mathbf{0 . 8} \mathbf{p p m}$ \\
\hline 1 & Aspergillus Niger & $2.5 \pm 0.6$ & $2.1 \pm 0.4$ & $1.4 \pm 0.08$ \\
\hline 2 & Aspergillus flavus & $4.2 \pm 0.9$ & $3.5 \pm 0.7$ & $1.6 \pm 0.5$ \\
\hline 3 & Rhizopus stolonifer & $3.1 \pm 0.9$ & $2.4 \pm 0.6$ & $2.2 \pm 0.3$ \\
\hline 4 & Fusarium oxysporium & $4.0 \pm 0.9$ & $3.7 \pm 0.8$ & $3.0 \pm 0.5$ \\
\hline 5 & Meyerozyma caribbica & $2.2 \pm 0.7$ & $2.0 \pm 0.1$ & $1.5 \pm 0.08$ \\
\hline 6 & Hypocrea koningii & $3.6 \pm 0.9$ & $2.9 \pm 0.5$ & $2.3 \pm 0.1$ \\
\hline
\end{tabular}
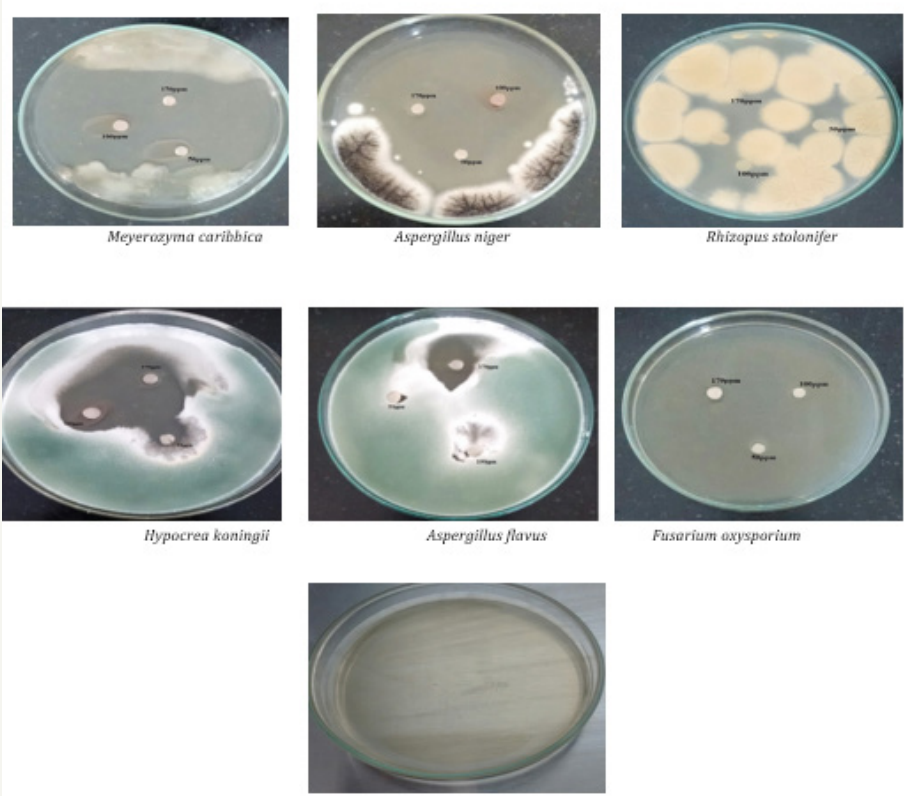

Fusarium axysporium

Control

Figure 8: Showing anti-fungal activity of Vitis vinifera peel extract mediated synthesized AgNPs.

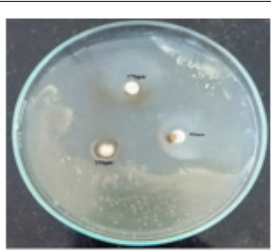

Bacillus pantothenticus
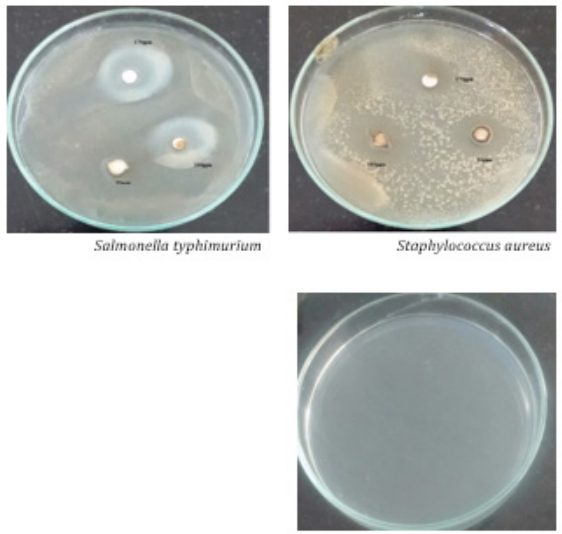

Control
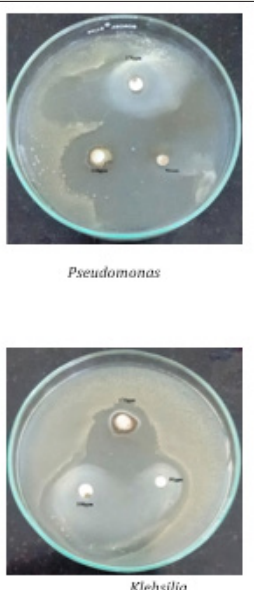

Pseudomonos

Klebsilio

Figure 9: Showing anti-bacterial activity of Vitis vinifera peel extract mediated synthesized AgNPs. 
The formations of free radicals from the surface of the silver nanoparticles were responsible for the antibacterial function. In addition, excess formation of reactive oxygen species (ROS) may lead to a breakdown of membrane function and increased permeability of the cell membrane or leakage of cell matters and morphological changes of bacterial cells and growth inhibition. The charge of bacterial cell wall is negative because of dissociation of carboxylic groups on the cell surface. Weak positive charges present on AgNPs are attracted towards negative charges. In contrast, Sondi and Salopek-Sondi suggested that the antibacterial effects of AgNPs on bacteria depended on the concentration of AgNPs and closely related with the development of "pits" on cell wall of bacteria. AgNPs interact with the thiol groups of bacterial proteins and may retard the replication of DNA. It is consistent to state that binding of the nanoparticles to the bacteria depends on the surface area available for interaction. Nanoparticles have larger surface area available for interaction which enhances bactericidal effect than the large sized particles; hence AgNPs exhibit more toxicity to the microorganism [8].

\section{Conclusion}

The present study concludes that Vitis vinifera peel extract can be used as source for synthesis of silver nanoparticles extracellularly. The biosynthetic methods have been recognized as an alternative to chemical and physical synthesis, as this biosynthetic method is economical, ecofriendly, and cost-effective. The present work exhibited an efficient and low-cost biological approach to synthesize the metal nanoparticles and provided helpful insight into the development of new antimicrobial agents with the synergistic enhancement of the antimicrobial mechanism against pathogenic microorganisms. The spectroscopic characterization data such as UV-Vis, FT-IR, XRD, DLS, TEM and SEM also support the formation and stability of the bio-synthesized AgNPs and this type of NPs used for medical applications for drugs in future

\section{Acknowledgement}

Authors are very much thankful to Acharya N G Ranga Agricultural University Tirupathi for carrying, characterization (UV, FT-IR, DLS) and supporting of this work.

\section{References}

1. Supraja N, Prasad TNVKV, Giridhara Krishna T (2016) Synthesis, characterization, and evaluation of the antimicrobial efficacy of Boswellia ovalifoliolata stem bark-extract-mediated zinc oxide nanoparticles. Appl Nanosci 6(4): 581-590

2. Korbekandi H, Iravani S (2012) Silver nanoparticles, the delivery of nanoparticles. In: Hashim Abbass A (Ed.), ISBN: 978-953- 51-0615-9, InTech, Iran.

3. Mohanpuria P, Rana NK, Yadav SK (2008) Biosynthesis of nanoparticles technological concepts and future applications. J Nanopart Res 10(3): 507-517.

4. Prabha S, Supraja N, Garud M, Prasad TNVKN (2014) Synthesis, characterization and antimicrobial activity of Alstonia scholaris barkextract-mediated silver nanoparticles. J Nanostruct. Chem 4(4): 161170.

5. Supraja N, Prasad TNVKN, Giridhara Krishna T, David E (2015) Synthesis, characterization, and evaluation of the antimicrobial efficacy of Boswellia ovalifoliolata stem bark-extract-mediated zinc oxide nanoparticles. Appl Nanosci 6(4): 581-590.

6. Supraja N, Prasad TNVKV, Dhanesh Gandhi A, Devipriya A, Kavitha P et al. (2018) Synthesis, characterization and evaluation of antimicrobial efficacy and brine shrimp lethality assay of Alstonia scholaris stem bark extract mediated ZnONPs. Biochemistry and Biophysics Reports 14: 6977.

7. Prasad TNVKV, Sudhakar P, SreenivasuluY, Latha P, Munaswamy V, et al. (2012) Effect of nanoscale zinc oxide particles on the germination, growth and yield of peanut. J Plant Nutr 35: 905-927.

8. Kayal Vizhi D, Supraja N, Devipriya A, et al. (2016) Evaluation of antibacterial activity and cytotoxic effects of green AgNPs against breast cancer cells (MCF 7). Adv Nano Res 4: 129-143.
Creative Commons Attribution 4.0 International License

For possible submissions Click Here
Submit Article

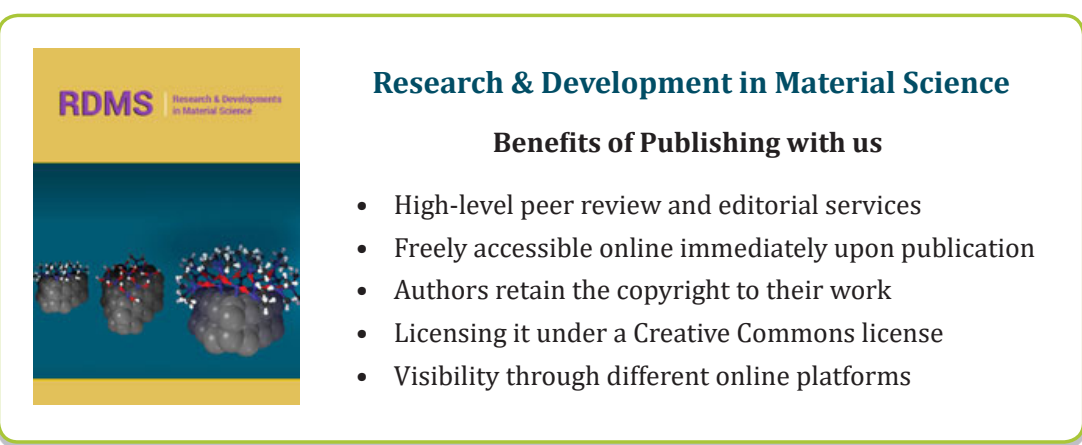

